\title{
Spoken language identification using i-vectors, $x$-vectors, PLDA and logistic regression
}

\author{
Ahmad Iqbal Abdurrahman, Amalia Zahra \\ Computer Science Department, Bina Nusantara University, Indonesia
}

\begin{tabular}{l}
\hline Article Info \\
\hline Article history: \\
Received Feb 19, 2021 \\
Revised May 20, 2021 \\
Accepted Jun 22, 2021 \\
\hline
\end{tabular}

\section{Keywords:}

Equal error rate

I-vector

Logistic regression

PLDA

Spoken language recognition

$\mathrm{X}$-vector

\begin{abstract}
In this paper, $\mathrm{i}$-vector and $\mathrm{x}$-vector is used to extract the features from speech signal from local Indonesia languages, namely Javanese, Sundanese and Minang languages to help classifier identify the language spoken by the speaker. Probabilistic linear discriminant analysis (PLDA) are used as the baseline classifier and logistic regression technique are used because of prior studies showing logistic regression has better performance than PLDA for classifying speech data. Once these features are extracted. The feature is going to be classified using the classifier mentioned before. In the experiment, we tried to segment the test data to three segment such as 3, 10, and 30 seconds. This study is expanded by testing multiple parameters on the i-vector and $\mathrm{x}$ vector method then comparing PLDA and logistic regression performance as its classifier. The $\mathrm{x}$-vector has better score than i-vector for every segmented data while using PLDA as its classifier, except where the i-vector and $\mathrm{x}$-vector is using logistic regression, i-vector still has better accuracy compared to $\mathrm{x}$ vector.
\end{abstract}

This is an open access article under the CC BY-SA license.

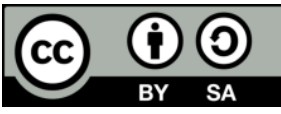

\section{Corresponding Author:}

Ahmad Iqbal Abdurrahman

Computer Science Department

Bina Nusantara University

Jakarta, Indonesia 11480

Email: ahmad.abdurrahman@binus.ac.id

\section{INTRODUCTION}

The number of regional languages in Indonesia that is recorded by Ministry of education and culture's national agency for language development and books is 652 languages. There is one language to bridge every language are called Bahasa Indonesia. This language is constructed from hundreds of languages that is used in Indonesia. But there are many Indonesian people that is not fluent at speaking Bahasa Indonesia. This matter can hinder people when they are speaking to the other. One example is if they are calling customer service and they are not using Bahasa Indonesia, the people in customer services may be experiencing difficulties when communicating with those people. Until now, there are 11 extinct regional languages. One of the reasons that language going extinct is, there are globalization effect, so people are learning global language like English for communicating with international people and using Bahasa Indonesia to communicate with domestic people. And they rarely used their own regional language.

Based on the problem written above, there is a technology called spoken language identification [1] to help people when they are visiting another region. This technology can help people by receiving input in the form of spoken sound, and then the system is going to select the matching language. These technology needs speech data for them to work. When collecting the dataset for this technology. It can also help to preserve regional language by archiving the dataset. 
With this technology, we can develop models that can recognize the language. If in the future a language translation system is made that can be integrated with the system in this study, regional language users can communicate directly with foreign language users when communicating. So that users can use their local language while using certain technologies, this will allow users to still use their own local language without fear of being affected by globalization.

There are several steps to identify language, starting from cleaning the data from noise to help the system get better accuracy, extracting the feature from speech data, and classifying the language. There are several techniques to classify the language spoken, including neural networks [2], convolutional neural networks [3]-[6], logistic regression [7], PLDA [8], gaussian mixture model [9], [10], support vector machine [11], [12], and several techniques to extract the features from the recording, such as MFCC [13], [14], ivector [15]-[17], and x-vector [18].

Jain, et al. [19] builds two systems, a system that uses the Mel-frequency cepstral coefficients (MFCC) and shifted delta cepstrum (SDC) features. At the time of testing, the result of a significant increase in accuracy on features, because MFCC performs better than SDC, therefore his research with MFCC. They use the support vector machine (SVM) classification technique combined with the singular value decomposition (SVD) technique to reduce its dimensions. This study produces a model with the best accuracy of $93.50 \%$ on a 30-minute pronunciation test with the callfriend corpus dataset (Martin and Przybocki, 2003) which has a total of twelve languages.

Venkatesan, et al. [20] used the MFCC technique which mimics parts of sound perception and production to get the details of the message uttered by the speaker. In this study, the dataset used has 4 languages, namely English, Hindi, Tamil, and Telugu, this sound file is sent to an extraction program that uses each sound chunk, then stored in CSV form. Acoustic models used for language identification are decision-tree classifier and SVM. This study produces $76 \%$ accuracy using SVM methods and $73 \%$ accuracy using decision tree classifier.

Sarthak, et al. [21] used raw audio waveforms as sound input which provide increased performance by avoiding overheads in calculating log-Mel spectrum for each audio file. This study uses the convolutional neural networks classification method because based on the journals studied, convolutional neural networks have very good performance compared to other machine learning techniques, the sound input data used is raw audio waveform because it is quite popular, because raw audio waveforms have advantages. That is, it can retrieve several features in the sound that cannot be extracted properly by several other feature extraction techniques such as MFCC, constant q transform (CQT), and fast fourier transform (FFT). In this study, 3 models were made, namely 1D ConvNet, 2D ConvNet, 2D ConvNet with Attention and GRU. 1D ConvNet has a hyper parameter random search algorithm that is useful for finding the optimal group of hyper parameters based on the given parameters. 2D ConvNet with Attention and bi-directional GRU models are added with a residual connection at each convolutional layer. This function is to find the optimal number of layers to train and eliminate the problem of vanishing gradients, there is also additional spatial attention that serves to help the model focus more on a specific part of the image, and this model also adds channel attention to help the model look for inter- dependencies between color channels in the log-Mel spectra. ConvNet 2D model explained that this model is like 2D ConvNet model with Attention and bi-directional GRU, but this model does not have the skip connection, attention layers, bi-directional GRU, and layers that were in the previous model. This study produces $93.7 \%$ accuracy using 1D ConvNet, $95.4 \%$ accuracy using 2D ConvNet, $95.0 \%$ accuracy using 2D ConvNet-Bidirectional + GRUAttention, and 96.6 accuracy from 2D ConvNet alone.

Heracleous, et al. [22] uses feature extraction i-vector techniques for language identification. The research was conducted using three classification techniques, namely deep neural network (DNN), convolutional neural network (CNN) and support vector machines (SVM). The dataset used in this study is NIST LRE 2015 (NIST, 2015) which has 50 languages to classify. Their system has a score of 1.89 EER from DNN technique. 2.00 EER from CNN technique, and 2.42 EER from SVM techniques.

Safitri, et al. [23] using the phone recognition method followed by language modeling (PRLM) and parallel phone recognition followed by language modeling (PPRLM) to identify the three selected languages. The difference between the two methods is the number of telephone identifiers used, if PRLM only uses one telephone identifier, PPRLM can use more than one telephone identifier for the language classification process. In this study, three languages were obtained with an average total sound duration per language of about 244 minutes. The voice data used are recordings of speakers reading scripts in their local language. The script contains articles or short stories in their local language. The experimental results compared were accuracy, false positive values, precision, and repeatability. PRLM experiments were carried out by trying each language module in PRLM. The existing modules start from Czech, English, Hungarian, and Spanish. Then the PPRLM experiment was carried out using all available language models and selecting the two 
language modules that had the best value in the PRLM experiment. This study produced average accuracy of 73.92\% from PPRLM techniques, and 67.04\% from PRLM techniques.

Snyder, et al. [18] conducted research on the x-vector feature extraction technique, the study compared the $\mathrm{x}$-vector technique with the $\mathrm{i}$-vector technique. This research also compared the performance of acoustic features and two types of bottleneck features. For acoustic features, 23 MFCC will be used, while for the bottleneck feature, fisher English BNFs and multilingual BNFs will be used. The classification is performed by a discriminatively trained gaussian classifier, the dataset used in this study is the dataset obtained from NIST LRE 2017 (NIST, 2017) which has five language groups. In this study, the EER score was 0.163 for the $\mathrm{x}$-vector system, and an EER score of 0.189 for i-vector.

Martinez, et al. [24] conducted research on the i-vector extraction method with the SVM classification method and logistic regression. In this study, the logistic regression accuracy score was obtained which was slightly higher than the SVM method at 3 sound durations $(3,10,30)$ seconds on the NIST LRE 2009 dataset which has 23 languages. Models that use SVM as classifier has 400 dimensions i-vector, while models that use logistic regression have 600 dimensions i-vector. The logistic regression technique has a better accuracy on every dataset duration $(3,10,30$ seconds).

Burget, et al. [25] conducted research using i-vector as its feature extraction, and used four classifier methods namely PLDA, LR, SVM, and havy-tailed PLDA, on this research it is shown that the LR method has better accuracy than the baseline PLDA method. I-vector is state of the art technique for spoken language recognition, but from the previous study [18], x-vector has better score than i-vector for classifying spoken language. The previous study [18] does not review how x-vector compared to i-vector technique using only MFCC as its acoustic feature in segmented duration while using classifier other than PLDA. From the research in [25], it is shown that LR has better accuracy than PLDA, so this experiment will compare logistic regression performance against PLDA as classifier. The current study focuses on spoken language identification using i-vector and $\mathrm{x}$-vector techniques as their feature extraction method. And comparing PLDA and logistic regression classifier performance to classify the language after the dataset feature has been extracted by the help of $\mathrm{i}$-vector and $\mathrm{x}$-vector. The current study also expanded by testing the dataset on segmented duration such as 3, 10, and 30 seconds of test data, finding best parameter for each extraction techniques, and comparing EER score for each classification techniques.

\section{RESEARCH METHOD}

\subsection{Dataset}

This study will use three regional languages, Bahasa Sunda, Bahasa Jawa, and Bahasa Minang. Data collection will be done by searching for sound samples of each language via the internet. For Javanese and Sundanese languages, it will be found on the openslr.org site. Table 1 describes the Javanese, Sundanese and Minangkabau language dataset used for training and testing the models.

Table 1. Dataset duration

\begin{tabular}{lc}
\hline Language & Duration \\
\hline Jawa & 200 Minutes \\
Sunda & 200 Minutes \\
Minang & 200 Minutes \\
\hline
\end{tabular}

The Minang language dataset will be collected via video from the YouTube site and pre-processing will be carried out such as changing the format of the speech data into WAV extension format and using $128 \mathrm{kbps}$ bitrate.

\subsection{Pre-processing}

After getting the Minang language dataset. Each of them will be combined to 200 minutes of recording. And then the dataset will be split by 10 seconds for each file. The dataset is separated by a ratio of $8: 1: 1$, with $80 \%$ as the data used for the training models, $10 \%$ as data for validation set, and $10 \%$ as data to be used for classification testing. After the dataset is divided by the ratio 80:10:10, which is 160 minutes for learning, 20 minutes for validation set, and 20 minutes for testing. The testing and validation dataset will be divided again into 3 parts, namely the 3,10 , and 30 second dataset.

\subsection{Model development}

This research will use $\mathrm{i}$-vector and $\mathrm{x}$-vector feature extraction technique to compare the accuracy results of the two. Both techniques used the same dataset so that the accuracy can be compared through the 
PLDA and logistic regression classifier. Before being fed to the i-vector or $\mathrm{x}$-vector techniques, the features are mean-normalized over a 3 -seconds sliding window, and nonspeech frames are removed using energybased speech activity. The acoustic features used in this study are 23 MFCC with a frame-length of $25 \mathrm{~ms}$.

The i-vector are built from GMM-UBM recipe described in [18] as the baseline system. The i-vector UBM size and i-vector dimension parameter will be tuned. The UBM parameter has a 2048, 1024, and 512 component full-covariance GMM. The i-vector has a dimension of 600, 400, 200 and iterated 2 times on the train dataset. The $\mathrm{x}$-vector training examples are built by picking speech chunks from the train data that are 2-5 seconds long for 5 epochs. The minibatch size used are 128. The parameters that will be tuned in the $\mathrm{x}$-vector technique are max-frame-chunk-size and num-repeats. Max-frame-chunk-size used in this research are 400, 500, or 600. The num-repeats value for the x-vectors model are 300, 600 or 900 .

Classifier models will be created using PLDA and logistic regression, before the dataset got classified using PLDA, the embeddings are whitened, length normalized, and gets their dimensionality reduced to 100,200 , or 300 using LDA. The training recordings are built from series of 10 seconds of speech.

\subsection{Evaluation}

In this study, the equal error rate (EER) score for each parameter will be measured. The models are built to predict each language. The experiment will be divided to two section, tuning the parameter for each model using validation dataset, and testing the final model using test set. After obtaining the results of the evaluation, an analysis of the results will be carried out, then the results of the analysis will answer the problem formulation.

\section{RESULTS AND DISCUSSION}

The experiment is focused on comparing performance between $\mathrm{i}$-vector and $\mathrm{x}$-vector techniques on three segmented durations. We performed the classification on three different languages. Once the models are trained and the feature are extracted, we used PLDA or logistic regression classifier to classify the dataset. The accuracy score of classification between three languages are reported in terms of EER. Tuning the i-vector parameter using validation dataset are recorded on Table 2.

Table 2. I-vector parameter score using PLDA classifier

\begin{tabular}{ccccc}
\hline \multirow{2}{*}{ UBM Size } & \multirow{2}{*}{ I-Vector Dimension } & \multicolumn{3}{c}{ EER Score using LDA+PLDA } \\
\cline { 3 - 5 } & & $3 \mathrm{~s}$ & $10 \mathrm{~s}$ & $30 \mathrm{~s}$ \\
\hline 2048 & 600 & 19.5 & 14.17 & 8.333 \\
1024 & 600 & 18.17 & 10.83 & 2.5 \\
512 & 600 & 15.67 & 6.111 & 0.8333 \\
512 & 200 & 16.75 & 8.661 & 1.667 \\
512 & 400 & 15.25 & 6.667 & 0.8333 \\
512 & 600 & 15.67 & 6.111 & 0.8333 \\
\hline
\end{tabular}

In Table 2, we see that smaller UBM Size has better accuracy, while the best UBM size is 512, the ivector dimension has different accuracy. On 3 seconds, the 400 dimensions i-vector has better accuracy than the 600 dimensions, while on the 10 seconds dataset, the 600 dimension has better accuracy. Not only smaller UBM Size has better accuracy, but it also has faster training time. And next, the LDA dimension parameter will be tuned, using 512 UBM size and 600 i-vector dimensions, the score is shown on Figure 1.

In Figure 1, it is shown that applying smaller LDA value has better EER score on every segment. Model with 100 LDA has best accuracy with 14.42 EER on $3 \mathrm{sec}, 4.444$ EER on $10 \mathrm{sec}$, and 0.833 on $30 \mathrm{sec}$ dataset. Next the model is tuned using the same parameter but using logistic regression as its classifier technique, the result is shown at Table 3.

In Table 3 we see very good score when using logistic regression compared to PLDA. Smaller UBM Size parameter still has big impact on the accuracy when using logistic regression. While the i-vector dimension does not affect the EER score as big as the UBM Size parameter tuning. On the same parameter, it has a much better score than the i-vector model that uses PLDA as its classifier.

After getting a score with the $\mathrm{i}$-vector technique, this study will review the score of the $\mathrm{x}$-vector using PLDA as the classifier using parameters previously described. The results using $\mathrm{x}$-vector technique are written on Table 4. 


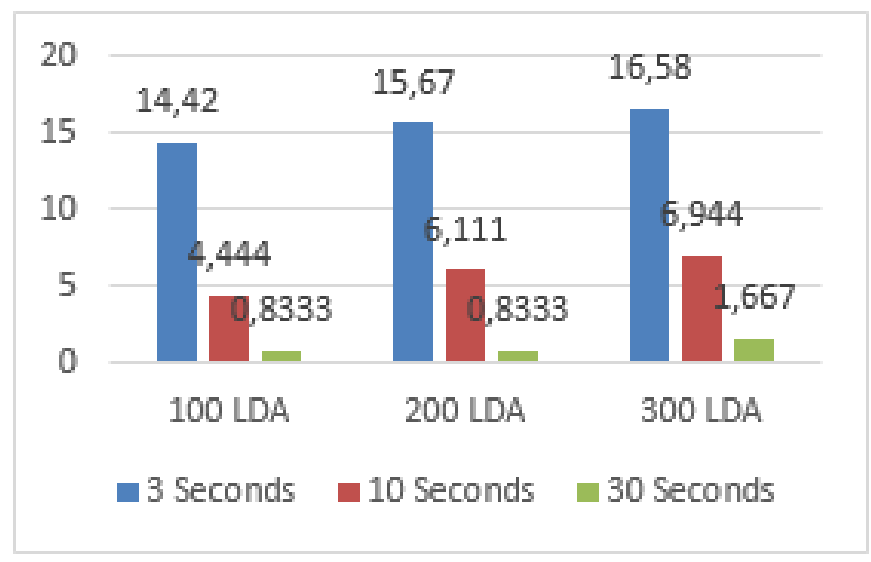

Figure 1. LDA dimension score comparation on i-vector model

At Figure 1, it is shown that applying smaller LDA value has better EER score on every segment. Model with 100 LDA has best accuracy with 14.42 EER on $3 \mathrm{sec}, 4.444$ EER on $10 \mathrm{sec}$, and 0.833 on $30 \mathrm{sec}$ dataset. Next the model is tuned using the same parameter but using logistic regression as its classifier technique, the result is shown at Table 3 .

Table 3. I-vector parameter score using logistic regression classifier

\begin{tabular}{ccccc}
\hline \multirow{2}{*}{ UBM Size } & \multirow{2}{*}{ I-Vector Dimension } & \multicolumn{3}{c}{ EER Score Using Logistic Regression } \\
\cline { 3 - 5 } & & $3 \mathrm{~s}$ & $10 \mathrm{~s}$ & $30 \mathrm{~s}$ \\
\hline 2048 & 600 & 13.75 & 6.944 & 4.167 \\
1024 & 600 & 9.5 & 5 & 1.67 \\
512 & 600 & 8.17 & 2.22 & 0 \\
512 & 200 & 9.25 & 2.222 & 0 \\
512 & 400 & 8.25 & 1.667 & 0 \\
512 & 600 & 8 & 1.39 & 0 \\
\hline
\end{tabular}

In table 3 we see very good score when using logistic regression compared to PLDA. Smaller UBM Size parameter still has big impact on the accuracy when using logistic regression. While the i-vector dimension does not affect the EER score as big as the UBM Size parameter tuning. On the same parameter, it has a much better score than the i-vector model that uses PLDA as its classifier.

After getting a score with the i-vector technique, this study will review the score of the $\mathrm{x}$-vector using PLDA as the classifier using parameters previously described. The results using $\mathrm{x}$-vector technique are written on Table 4.

Table 4. X-vector parameter score using PLDA classifier

\begin{tabular}{ccccc}
\hline \multirow{2}{*}{ Max-frame-chunk-size } & \multirow{2}{*}{ Num-repeats } & \multicolumn{3}{c}{ EER Score using LDA+PLDA } \\
\cline { 3 - 5 } & & $3 \mathrm{~s}$ & $10 \mathrm{~s}$ & $30 \mathrm{~s}$ \\
\hline 400 & 300 & 16.25 & 10.56 & 5.833 \\
500 & 300 & 14.67 & 5.833 & 1.667 \\
600 & 300 & 16.42 & 5.833 & 1.667 \\
500 & 300 & 14.83 & 5.833 & 1.667 \\
500 & 600 & 13.25 & 5.733 & 0.8333 \\
500 & 900 & 10.83 & 3.056 & 0 \\
\hline
\end{tabular}

In Table 4, 500 max-frame-chunk-size has the best accuracy on 3, 10, and 30s dataset, this means that putting high value on this parameter does not get the accuracy to get better. The num-repeats tuning used 500 as its max-frame-chunk-size. Changing the num-repeats value to higher value will increase the amount of training time for the models, it takes nearly three times longer training time when using 900 num-repeats compared to 300 num-repeats. Next, using the best parameter from the Table 4, the LDA parameter will be tuned for $\mathrm{x}$-vector technique. The results are shown on Figure 2. 


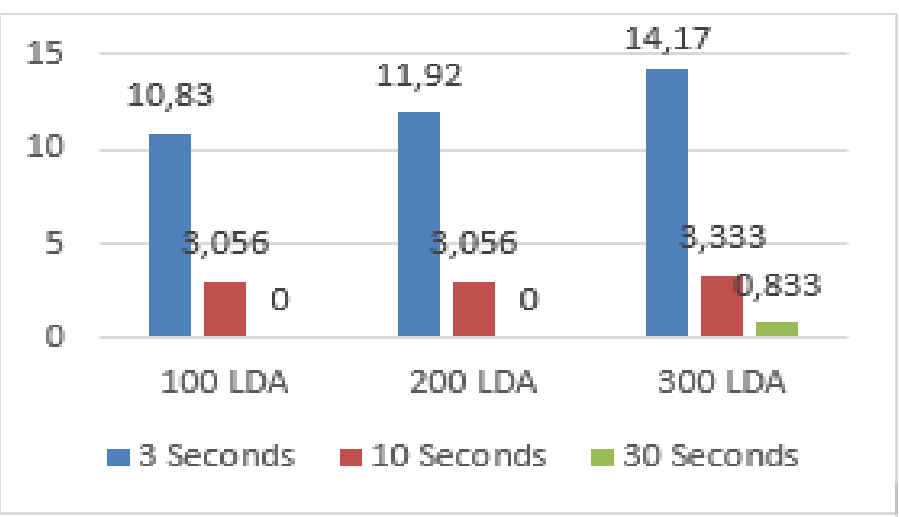

Figure 2. LDA dimension score comparation on $\mathrm{x}$-vector model

Judging from i-vector score using PLDA classifier, $x$-vector has better accuracy on validation set when using smaller LDA. Getting EER of 10.83 on 3 seconds, 3.056 on 10 seconds, and 0 on 30 seconds. After reviewing $\mathrm{x}$-vector using PLDA classifier, now we will review $\mathrm{x}$-vector performance using logistic regression classifier using the same parameter as PLDA model, the results are shown on Table 5.

Table 5. X-vector parameter score using logistic regression classifier

\begin{tabular}{ccccc}
\hline \multirow{2}{*}{ Max-frame-chunk-size } & \multirow{2}{*}{ Num-repeats } & \multicolumn{3}{c}{ EER Score using Logistic Regression } \\
\cline { 3 - 5 } & & $3 \mathrm{~s}$ & $10 \mathrm{~s}$ & $30 \mathrm{~s}$ \\
\hline 400 & 300 & 15.08 & 5.833 & 3.333 \\
500 & 300 & 14.83 & 5.278 & 1.667 \\
600 & 300 & 15.75 & 8.333 & 5 \\
500 & 300 & 14.67 & 5.278 & 1.667 \\
500 & 600 & 8.667 & 1.667 & 0 \\
500 & 900 & 9.083 & 2.5 & 0 \\
\hline
\end{tabular}

From the score on Table 5, just like i-vector, it is shown that $\mathrm{x}$-vector has better score when using logistic regression as its classifier. In the next section, we compare i-vector and x-vector with PLDA or logistic regression using test set. Parameter used in this section are parameter that has the best score on section before. The results for this comparation are shown on Table 6.

Table 6. Using best parameter on test set

\begin{tabular}{cccc}
\hline Technique & $3 \mathrm{~s}$ & $10 \mathrm{~s}$ & $30 \mathrm{~s}$ \\
\hline I-Vector + PLDA & 13.17 & 3.333 & 0 \\
X-Vector + PLDA & 10.5 & 2.778 & 0 \\
I-Vector + LR & 6 & 0.8333 & 0 \\
X-Vector + LR & 7.5 & 0.8333 & 0 \\
\hline
\end{tabular}

From the Table 6, It can be concluded, i-vector and x-vector has better accuracy when using logistic regression compared to PLDA classifier. But i-vector still has better accuracy than $\mathrm{x}$-vector when using logistic regression as its classifier. Next, we try to get the EER from each language, so we can see how these models perform for each language. The results for each model on each language is shown on Table 7.

Table 7. Models EER score for each language

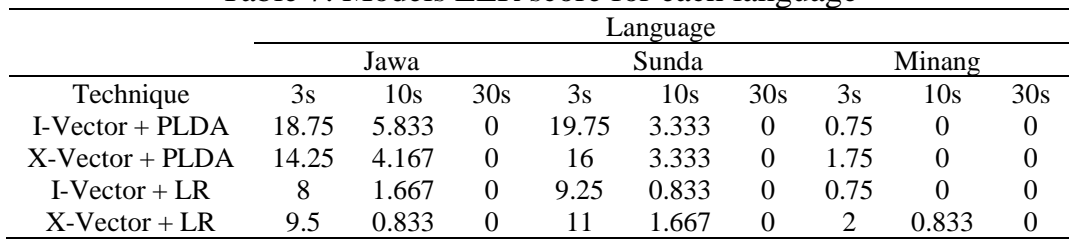


In Table 7 we see Jawa and Sunda language has similar score for each duration, and Minang has the best accuracy from these three languages. While Minang language has so much better EER than the other two languages, this can be caused by Minang recordings are made of several long videos that have been split into 3, 10 and 30 second segments, so that many files have similar recording quality. These models have no problem when classifying language that has long duration like 30 seconds and has very good accuracy when classifying 10 seconds segment. It is still having a bit difficulty when classifying the 3 seconds recording, but $\mathrm{x}$-vector still has the edge over $\mathrm{i}$-vector when classifying the short recordings while using PLDA as its classification technique.

\section{CONCLUSION}

In this paper, we compared i-vector and $\mathrm{x}$-vector for automatic language identification system in Kaldi to get which parameter has better accuracy for each technique and which feature extraction techniques perform better using PLDA or LR method as its classifier. The features were extracted from audio recording to form a training set using $\mathrm{i}$-vector and $\mathrm{x}$-vector technique and using PLDA or logistic regression technique to identify the language spoken. X-vector has the best score in every segment using PLDA classifier. But $\mathrm{i}$-vector still has better accuracy compared to $\mathrm{X}$-vector when using logistic regression as its classifier. When using $\mathrm{i}$-vector and $\mathrm{x}$-vector, it is shown that logistic regression please change more detailshas performed better than PLDA as classifier. This experiment shows that using LR as classifier was a good decision to help $\mathrm{x}$-vector performs better. Our suggestion for the future research is, to get more recording duration and variation for Minang language, trying other acoustic feature such as bottleneck feature, using other classifier methods or combining them, and getting other local language to be classified.

\section{ACKNOWLEDGEMENTS}

The authors would like to express sincere thanks to the Bina Nusantara University for supporting this research, and thanks to Mrs. Amalia Zahra from University of Bina Nusantara for the guidance, very useful discussion, and the assistance for this research. Without her support and guidance, this research would not have been possible, the assistance provided was greatly appreciated.

\section{REFERENCES}

[1] H. Li, B. Ma, and K. A. Lee, "Spoken Language Recognition: From Fundamentals to Practice," in Proceedings of the IEEE, vol. 101, no. 5, pp. 1136-1159, May 2013, doi: 10.1109/JPROC.2012.2237151.

[2] G. Montavon, "Deep learning for spoken language identification," In NIPS Workshop on deep learning for speech recognition and related applications, pp. 1-4, 2009.

[3] S. Mukherjee, N. Shivam, A. Gangwal, L. Khaitan, and A. J. Das, "Spoken Language Recognition Using CNN," 2019 International Conference on Information Technology (ICIT), 2019, pp. 37-41, doi: 10.1109/ICIT48102.2019.00013.

[4] L. Ferrer, Y. Lei, M. McLaren, and N. Scheffer, "Spoken language recognition based on senone posteriors," in Proceedings of the Annual Conference of the International Speech Communication Association, INTERSPEECH, 2014, pp. 2150-2154.

[5] C. Korkut, A. Haznedaroğlu, and L. M. Arslan, "Spoken Language Identification with Deep Convolutional Neural Network and Data Augmentation," 2020 28th Signal Processing and Communications Applications Conference (SIU), 2020, pp. 1-4, doi: 10.1109/SIU49456.2020.9302425.

[6] R. Ubale, Y. Qian, and K. Evanini, "Exploring End-To-End Attention-Based Neural Networks For Native Language Identification," 2018 IEEE Spoken Language Technology Workshop (SLT), 2018, pp. 84-91, doi: 10.1109/SLT.2018.8639689.

[7] D. A. Van Leeuwen, and N. Brummer, "Channel-dependent GMM and Multi-class Logistic Regression models for language recognition," 2006 IEEE Odyssey - The Speaker and Language Recognition Workshop, San Juan, PR, USA, 2006, pp. 1-8, doi: 10.1109/ODYSSEY.2006.248094.

[8] M. K. Rai, Neetish, M. S. Fahad, J. Yadav, and K. S. Rao, "Language identification using PLDA based on i-vector in noisy environment," 2016 International Conference on Advances in Computing, Communications and Informatics (ICACCI), 2016, pp. 1014-1020, doi: 10.1109/ICACCI.2016.7732177.

[9] A. Dustor and P. Szwarc, "Spoken language identification based on GMM models," ICSES 2010 International Conference on Signals and Electronic Circuits, 2010, pp. 105-108.

[10] Y. Xu, J. Yang, and J. Chen, "Methods to Improve Gaussian Mixture Model for Language Identification," 2010 International Conference on Measuring Technology and Mechatronics Automation, 2010, pp. 656-659, doi: 10.1109/ICMTMA.2010.545.

[11] S. Mohanty, "Phonotactic Model for Spoken Language Identification in Indian Language Perspective." International Journal of Computer Applications, 2011 .pp 18-24. 10.5120/2389-3164.

[12] R. W. M. Ng, T. Lee, C. Leung, B. Ma, and H. Li, "Spoken Language Recognition With Prosodic Features," in IEEE Transactions on Audio, Speech, and Language Processing, vol. 21, no. 9, pp. 1841-1853, Sept. 2013, doi: 
10.1109/TASL.2013.2260157.

[13] L. Ferrer, "Joint probabilistic linear discriminant analysis," arXiv preprint arXiv:1704.02346, 2017.

[14] T. S. Gunawan, R. Husain, and M. Kartiwi, "Development of language identification system using MFCC and vector quantization," 2017 IEEE 4th International Conference on Smart Instrumentation, Measurement and Application (ICSIMA), 2017, pp. 1-4, doi: 10.1109/ICSIMA.2017.8312034.

[15] N. Dehak, P. Torres-Carrasquillo, D. Reynolds, and R. Dehak. "Language Recognition via i-vectors and Dimensionality Reduction," INTERSPEECH, 2011, pp. 857-860.

[16] P. Heracleous, Y. Mohammad, K. Takai, K. Yasuda, and A. Yoneyama, "Spoken Language Identification Based on I-vectors and Conditional Random Fields," 2018 14th International Wireless Communications \& Mobile Computing Conference (IWCMC), 2018, pp. 1443-1447, doi: 10.1109/IWCMC.2018.8450327.

[17] M. Li and L. Wenbo. "Speaker verification and spoken language identification using a generalized i-vector framework with phonetic tokenizations and tandem features," in Fifteenth Annual Conference of the International Speech Communication Association, 2014.

[18] D. Snyder, D. Garcia-Romero, A. McCree, G. Sell, D. Povey, and S. Khudanpur, "Spoken Language Recognition using X-vectors," Odyssey 2018 The Speaker and Language Recognition Workshop, pp. 105-111, 2018, doi: 10.21437/odyssey.2018-15.

[19] M. Jain, M. S. Saranya, and H. A. Murthy, "An SVD Based Approach for Spoken Language Identification," 2018 International Conference on Signal Processing and Communications (SPCOM), 2018, pp. 312-316, doi: 10.1109/SPCOM.2018.8724477.

[20] H. Venkatesan, T. V. Venkatasubramanian, and J. Sangeetha, "Automatic Language Identification using Machine learning Techniques," 2018 3rd International Conference on Communication and Electronics Systems (ICCES), 2018, pp. 583-588, doi: 10.1109/CESYS.2018.8724070.

[21] Sarthak, S. Shukla, and G. Mittal, "Spoken Language Identification Using ConvNets," Lecture Notes in Computer Science, pp. 252-265, 2019, doi: 10.1007/978-3-030-34255-5 17.

[22] P. Heracleous, K. Takai, K. Yasuda, Y. Mohammad, and A. Yoneyama, "Comparative Study on Spoken Language Identification Based on Deep Learning," 2018 26th European Signal Processing Conference (EUSIPCO), 2018, pp. 2265-2269, doi: 10.23919/EUSIPCO.2018.8553347.

[23] N. E. Safitri, A. Zahra, and M. Adriani. "Spoken language identification with phonotactics methods on minangkabau, sundanese, and javanese languages," Procedia Computer Science, vol. 81, pp. 182-187, 2016, doi: 10.1016/j.procs.2016.04.047.

[24] D. Martinez, O. Plchot, L. Burget, O. Glembek, P. Matejka, "Language Recognition in iVectors Space," INTERSPEECH, 2011, pp. 861-864.

[25] L. Burget, O. Plchot, S. Cumani, O. Glembek, P. Matějka and N. Brümmer, "Discriminatively trained Probabilistic Linear Discriminant Analysis for speaker verification," 2011 IEEE International Conference on Acoustics, Speech and Signal Processing (ICASSP), 2011, pp. 4832-4835, doi: 10.1109/ICASSP.2011.5947437.

\section{BIOGRAPHIES OF AUTHORS}

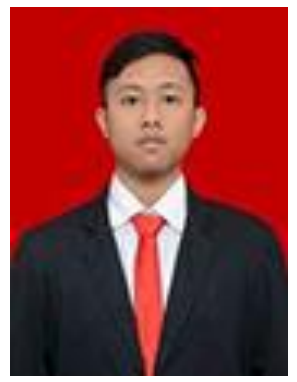

Ahmad Iqbal Abdurrahman is currently a graduate student in Computer Science Departement of Bina Nusantara University. He received his bachelor's degree in Information Technology from Bina Nusantara University in 2019. His research interest include speech technology such as Spoken Language Identification.

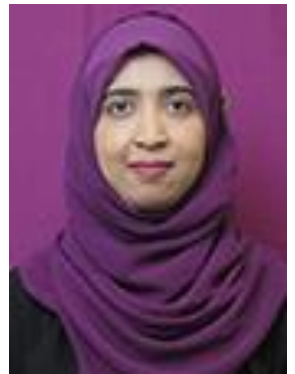

Amalia Zahra is a lecturer at the Master of Information Technology, Bina Nusantara University, Indonesia. She received her bachelor's degree in computer science from the Faculty of Computer Science, University of Indonesia (UI) in 2008. She does not have a master's degree. Her PhD was obtained from the School of Computer Science and Informatics, University College Dublin (UCD), Ireland in 2014. Her research interests cover various fields in speech technology, such as speech recognition, spoken language identification, speaker verification, speech emotion recognition, and so on. Additionally, she also has interest in Natural Language Processing (NLP), computational linguistics, machine learning, and artificial intelligence. 